\title{
Primary events in the photodissociation of oxyhemoglobin
}

\author{
Atsushi Yabushita ${ }^{a}$ and Takayoshi Kobayashi ${ }^{\text {a,b,c,d,* }}$ \\ ${ }^{a}$ Department of Electrophysics, National Chiao-Tung University, Hsichu, Taiwan \\ ${ }^{\mathrm{b}}$ ICORP, JST, Honcho, Kawaguchi, Saitama, Japan \\ ${ }^{\mathrm{c}}$ Department of Applied Physics and Chemistry and Institute for Laser Science, University of \\ Electro-Communications, Chofu, Tokyo, Japan \\ ${ }^{\mathrm{d}}$ Institute of Laser Engineering, Osaka University, Suita, Osaka, Japan
}

\begin{abstract}
The growing number of data on heme proteins in the ultrafast time domain have revealed complicated photophysics of the heme that are sensitive both to the structure of the protein and to the ligand species. In this work, ultrafast time-resolved pump-probe in visible spectral range was performed using an ultrashort visible laser pulse. Broad spectral width of visible laser pulse enabled us to observe the pump-probe signal with a broadband range. A broadband multi-channel detector array coupled to a multi-channel lock-in amplifier was used to obtain the pump-probe signals at all of the probe frequencies simultaneously. In the simultaneous measurement at many probe wavelengths, we could obtain ultrafast spectral change after the photo-excitation of oxy-hemoglobin only with a short measurement time, avoiding laser damage on the sample. The time constant of the primary process was determined for the first time. Ultrafast spectral change observed in the early delay time region directly shows ultrafast photo-dissociation process of oxy-hemoglobin.
\end{abstract}

Keywords: Ultrafast spectroscopy, hemoglobin, photo-dissociation

\section{Introduction}

Ultrafast laser experiment using ultra-short laser pulses can reveal the transient existence of intermediate species in the chemical reactions [22]. The ultrafast spectroscopy is a complementary method to those of electron diffraction method by Miller et al. [21] and X-ray diffraction methods by the groups of Anfinrud [23] and Zewail [24]. Instantaneous vibrational amplitude detection reaching sub-fs thanks to the pulse duration of $5 \mathrm{fs}$ and the high signal to noise ratio with high stability can provide much better time resolution than electron and X-ray diffraction experiments. Also the method can be used for the measurements of amorphous and liquid-phase materials, which may be difficult to be measured by $\mathrm{X}$-ray or electron diffraction. In our previous paper [11], we could even detect ultrafast real-time change in frequencies of in-plane and out-of-plane bending modes associated with the structural change during the photoisomerization of bacteriorhodopsin. Femtosecond stimulated Raman spectroscopy with high time and spectral resolution was applied to observe time-dependent conformational change [17], but the method cannot obtain the information of vibrational phase as is obtained in the ultrafast pump-probe spectroscopy.

\footnotetext{
${ }^{*}$ Corresponding author: Takayoshi Kobayashi, Department of Electrophysics, National Chiao-Tung University, Hsichu 300 , Taiwan. Tel.: +81 42443 5845; Fax: +81 42443 5825; E-mail: kobayashi@ils.uec.ac.jp.
} 
The growing body of data on heme proteins in the ultrafast time domain has revealed complicated photophysics of the heme that are sensitive to both the structure of the protein $[3,13-15,18]$ and to the ligand $\left(\mathrm{CO}, \mathrm{O}_{2}\right.$ and $\left.\mathrm{NO}\right)[3,4,8,10,13-16]$. Petrich et al. studied [20] the excited-state photophysics of hemoglobin $(\mathrm{Hb})$, myoglobin $(\mathrm{Mb})$, and protoheme and the effects of the ligands $\mathrm{CO}, \mathrm{O}_{2}$ and $\mathrm{NO}$ on the excited-state photophysics. Previous studies have demonstrated that heme photophysics is similar in hemoglobin $(\mathrm{Hb})$, heme proteins and heme model systems. All of these hemes exhibit two excited states designated with $\mathrm{Hb}_{\mathrm{I}}^{*}$ and $\mathrm{Hb}_{\mathrm{II}}^{*}[2,5-7,13,19,20]$. The data show that $\mathrm{Hb}_{\mathrm{I}}^{*}$ is formed in less than $50 \mathrm{fs}$ and then decays to form $\mathrm{Hb}_{\mathrm{II}}^{*}$ in $300 \mathrm{fs}$ [7]. The decay time of the $\mathrm{Hb}_{\mathrm{II}}^{*}$ electronic state to the ground state was about 3 ps. This electronic relaxation is so fast that intramolecular vibrational redistribution (IVR) and vibrational cooling occur on a similar (or even slightly longer) time scale. In spite of this energy difference, the electronic relaxation pathways of heme-CO and deoxy heme are thought to be similar [20]. Both the photodissociation of $\mathrm{CO}$ and the heme-iron movement out of plane occur very rapidly $(<50 \mathrm{fs})$ to create a species that resembles deoxy $\mathrm{Hb}$. Also, $\mathrm{Hb}_{\mathrm{I}}^{*}$ transient spectra appear on the same time scale as photolysis in both $\mathrm{Hb}^{*} \mathrm{CO}$ and $\mathrm{Hb} * \mathrm{NO}$ and the intensity and frequency of $\mathrm{Hb}_{\mathrm{I}}^{*}$ in deoxy $\mathrm{Hb}^{*}$ is the same as in photolyzed $\mathrm{Hb}^{*} \mathrm{CO}$ and $\mathrm{Hb} * \mathrm{NO}$. Although different in intensity, the similarity between the $\mathrm{Hb}_{\mathrm{II}}^{*}$ transient absorption bands in $\mathrm{Hb}^{*} \mathrm{CO}$ and deoxy $\mathrm{Hb}^{*}$ indicates that photophysical decay in both occurs by means of similar pathways. From the femtosecond transient absorption spectra and sub-picosecond (sub-ps) time-resolved resonance Raman spectra, the phenomena were explained by Franzen and others [7] in terms of the ultrafast electronic relaxation in the heme protein. A short-lived species $\mathrm{Hb}_{\mathrm{I}}^{*}$ was attributed to an iron-to-porphyrin ring charge transfer (CT) state. The $\mathrm{CT}$ character of $\mathrm{Hb}_{\mathrm{I}}^{*}$ can explain the ultrafast decay of this state, and provides a mechanism for photolysis. The proposed mechanism was claimed to explain the ultrafast intersystem crossing that has been proposed to result in photolysis $[9,25]$.

Above mentioned multiple electronic intermediate state model, which is based on single wavelength measurements [20], was considered not to adequately explain the observed broadband spectral dynamics by later experiment by Ye et al. in their careful study of photo-dissociation process of myoglobin [26,27]. Particularly observed spectral shift on the blue side of the Soret band by Ye et al. was considered to be better explained (unless even further more electronic states are postulated) by another model invoking hot photoproduct in the ground state. They demonstrated how their new model fully describes the ultrafast optical signals that occur in ferrous heme proteins. Moreover, ultrafast non-radiative electronic decay is consistent with the absence of detectable broadband luminescence from excited electronic states in heme systems. These results are consistent with an immediate (sub $100 \mathrm{fs}$ ) relaxation to the electronic ground state followed by vibrational equilibration. Relaxation of the hot photoproduct spectrum was well described using independent time scales for narrowing (about $400 \mathrm{fs}$ ) and blue shifting (0.4-4 ps) as the system returns to an equilibrium state.

In this work, ultrafast time-resolved pump-probe signal was observed using an ultrashort visible laser pulse in its broadband visible spectral range. The simultaneous measurement at as many as 128 probe wavelengths was performed using a multi-channel detector array to obtain ultrafast spectral change after the photo-excitation of oxy-hemoglobin. The simultaneous measurement can avoid laser damage in the sample. Analyzing the observed time traces, we determined lifetime components. The fastest time constant corresponds to the ultrafast photodissociation process of oxy-hemoglobin. The time constant of the primary process was determined to be $45 \pm 5$ fs for the first time. The spectrum of the fastest decay component was determined for the photo-excited oxy-hemoglobin in the spectral range of $719 \mathrm{~nm}$ 
$\left(13,914 \mathrm{~cm}^{-1}\right)$ and $523 \mathrm{~nm}\left(19,109 \mathrm{~cm}^{-1}\right)$ for the first time in the visible range. Ultrafast spectral change found in the measurement result is thought to be reflecting ultrafast photo-dissociation process of oxyhemoglobin.

\section{Experimental section}

Hemoglobin from bovine blood (Sigma-Aldrich) was dissolved in water with phosphate buffer stabilized at $\mathrm{pH}$ 7.7. Undissolved residue was removed by passing the solution through a filter (Millex; Millipore) put on a head of injection syringe. To prevent oxidization of the sample, sodium thiosulfate (Tokyo Chemical Industry, Ltd.) was added into the solution without further purification. The sample solution had an optical density of about 1.0 at $540 \mathrm{~nm}$ in a $1 \mathrm{~mm}$ cell (6210-27501; GL Science) used for femtosecond pump-probe experiment. The solutions were carefully prepared monitoring their absorption spectra by an ultraviolet-visible-near-infrared (UV-VIS-NIR) scanning spectrophotometer (UV-3101PC; Shimadzu).

A Ti:sapphire laser system (FemtoSource (sPRO); FemtoLasers) with an 8-path bow-tie amplifier (Femtopower; FemtoLasers) was used as a light source of pump-probe experiment. The width, energy, spectral range, and repetition rate of the pulses generated from the amplifier were $20 \mathrm{fs}, 30 \mu \mathrm{J}, 750$ $870 \mathrm{~nm}$ and $1 \mathrm{kHz}$, respectively. The output pulse was frequency doubled by a type-I $\beta$-barium borate (BBO) crystal and compressed using a prism pair to generate a $395 \mathrm{~nm}$ pulse having a width of $40 \mathrm{fs}$ at the $1 \mathrm{kHz}$ repetition rate. The frequency-doubled pulse was used as a pump pulse in this pump-probe measurement.

After the generation of frequency-doubled pulse in the BBO crystal, part of the fundamental pulse still remains in the light path of the pump beam without being frequency doubled. One of the prisms used for the compression of the pump beam displaced the residue of the fundamental pulse, which was also compressed up to 20 fs by passing through another prism pair. The compressed fundamental pulse was focused in a sapphire plate to generate visible broadband spectrum by causing self phase modulation in the sapphire plate. The visible broadband pulse was used as a probe pulse in the pumpprobe measurement.

Both of the pump and probe pulses were focused by a parabolic mirror into a $1 \mathrm{~mm}$ thick standard fluorimeter cells (6210-27501; GL Science). Time delay of the probe pulse with respect to the pump pulses was changed by an electrically driven stepper with a 1 fs step. The pump-probe signals were detected with a 128-channel lock-in amplifier. The experimental setup is shown in Fig. 1.

Observed stationary spectrum of sample has peaks at 540, 573 and $624 \mathrm{~nm}$. The locations of the peaks are in agreement with those in literature [1]. The spectrum of the laser pulse is extending from 520 to $720 \mathrm{~nm}$ with peak around $570 \mathrm{~nm}$ with a good overlap with the stationary absorption spectrum of the sample. All measurements were performed at room temperature $(294 \pm 1 \mathrm{~K})$.

\section{Results and discussion}

The laser spectrum overlaps well with the absorption spectrum of oxy-hemoglobin solution sample. The laser spectrum is composed of a main peak at $17,500 \mathrm{~cm}^{-1}$ and a weak peak around $14,500 \mathrm{~cm}^{-1}$ and a shoulder at about $16,000 \mathrm{~cm}^{-1}$. The absorption spectrum has two clearly separated peaks around 18,550 and $17,300 \mathrm{~cm}^{-1}$, which are attributed to $Q(00)$ and $Q(10)$, respectively. 


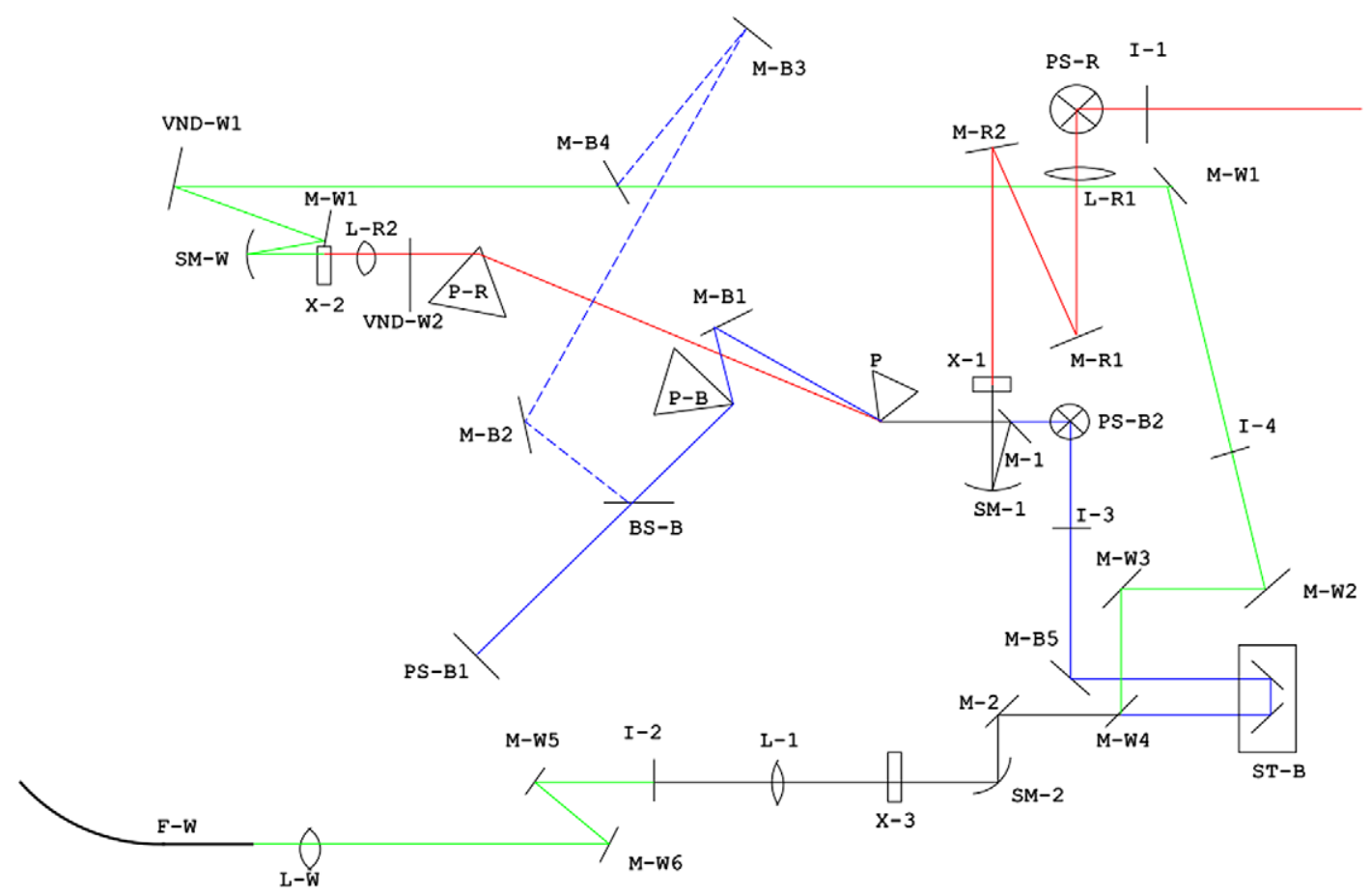

Fig. 1. Optical setup to generate pump and probe beams, and to perform pump-probe spectroscopy. I - iris; $\mathrm{L}$ - lens; $\mathrm{M}$ - mirror; $\mathrm{P}$ - prism; PS - periscope to rotate polarization in 90 degree; BS - beam splitter; SM - spherical mirror; ST - step scan stage to control probe delay time; $\mathrm{X}-1-\beta-\mathrm{BaB}_{2} \mathrm{O}_{3}$ (BBO) crystal for second harmonic generation; $\mathrm{X}-2$ - sapphire plate for white light generation; X-3 - BBO crystal or sample to be measured; F - fiber to couple light into polychromator connected to lock-in detector arrays.

The probe delay time dependence of absorbance change was observed in the time range extending from -70 to $1900 \mathrm{fs}$ at $1 \mathrm{fs}$ step. The sample is first excited to the $S_{2}$ state (the Soret band of heme) by the $395 \mathrm{~nm}$ pump pulse. The positive signal of the absorbance change observed in the visible region is considered to be the excited-state absorption from the $S_{2}$ state to a higher-lying excited state. Our previous work [12] shows how to classify dynamic vibronic couplings in vibrational real-time spectra. The probe wavelength dependency of Fourier intensity spectra could be discussed in the future work when vibrational phases of molecular vibrations are observed with good signal-to-noise ratio.

In the work by Ye et al. [26], they performed the time-resolved absorption measurement of myoglobin, both unligated and ligated with diatomic molecules. The pump wavelength was at $580 \mathrm{~nm}$, and the probe spectral range was near UV and blue spectral between 400 and $500 \mathrm{~nm}$. Their study on the photo-dissociation process of myoglobin demonstrated how their new model fully describes the ultrafast optical signals that occur in ferrous heme proteins. Moreover, ultrafast non-radiative electronic decay is consistent with the absence of detectable broadband luminescence from excited electronic states in heme systems. These results are consistent with an immediate (sub $100 \mathrm{fs}$ ) relaxation to the electronic ground state followed by vibrational equilibration. Relaxation of the hot photoproduct spectrum was well described using independent time scales for narrowing (about $400 \mathrm{fs}$ ) and blue shifting (0.4-4 ps) as the system returns to an equilibrium state.

We studied oxy-hemoglobin performing pump-probe measurement by ultrashort pulse to determine the shortest time constant, which corresponds to the ultrafast photo-dissociation process of oxy- 


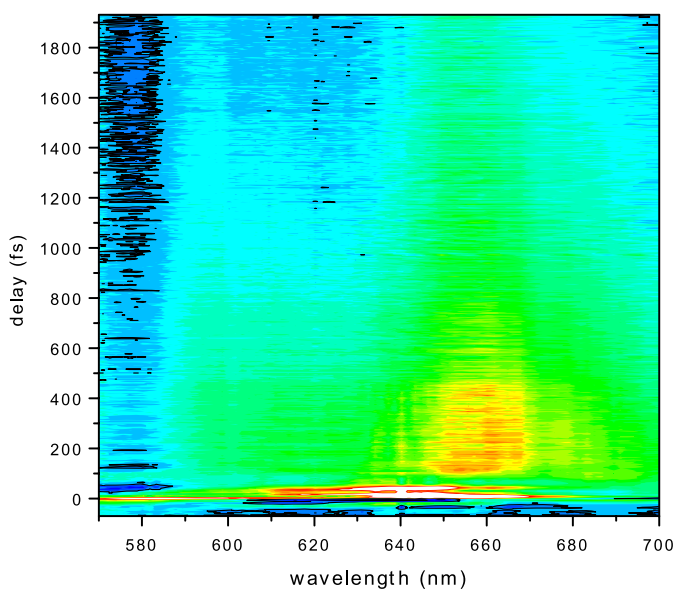

(a)

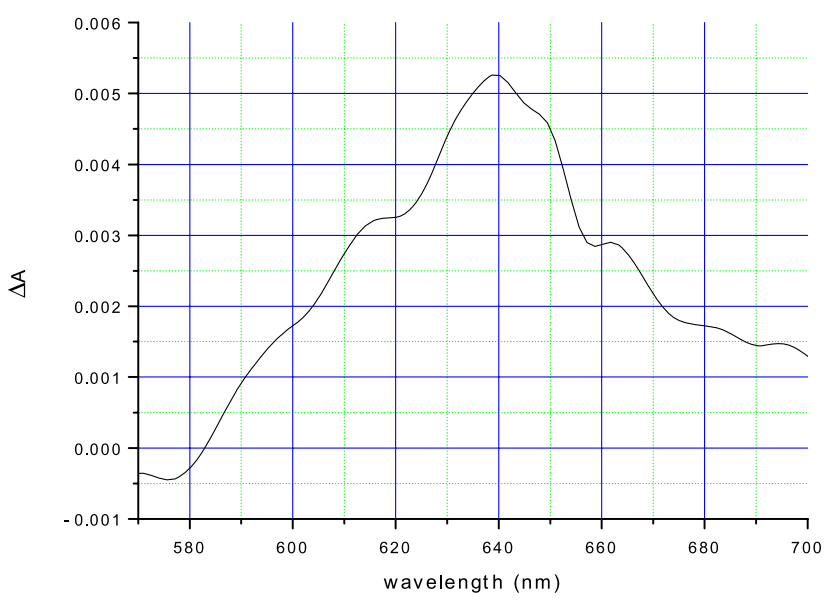

(b)

Fig. 2. (a) Two-dimensional display of time-resolved difference absorbance spectra. Black curves show contour of $\Delta A=0$. (b) Time-resolved difference absorbance spectrum averaged from 30 to $60 \mathrm{fs}$.

hemoglobin. Time-resolved difference absorption spectra were obtained in the delay range from -70 to $1930 \mathrm{fs}$ and probe spectral region from 523 to $719 \mathrm{~nm}$ as shown in Fig. 2(a). Figure 2(b) shows timeresolved difference absorbance spectrum at the earliest time-delay averaged from 30 to $60 \mathrm{fs}$. Obtained time traces were fitted by double exponential function in the probe wavelength region between 600 and $700 \mathrm{~nm}$ where $\Delta A$ is not close to zero. The uncertainty of the fitting results was estimated by the standard deviation of the obtained lifetimes. The reason why we choose the number of exponentials as two is because previous time-resolved studies of heme protein show that there are two lifetime components $\left(\tau_{1}<50 \mathrm{fs}\right.$ and $\tau_{2} \sim 1 \mathrm{ps}$ ) in the time region studied in this work. Exponential fitting of the observed time traces determined the two time constants. The fastest lifetime, $\tau_{1}=45 \pm 5 \mathrm{fs}$, corresponds to the ultrafast photo-dissociation process from the photo-excited $\mathrm{HbO}_{2}$ to the deoxy- $\mathrm{Hb}$, which is thought to be the vibrationally hot electronic ground state. The second shortest time constant $\tau_{2}=676 \pm 75 \mathrm{fs}$ is close to vibrational cooling time of about 1 ps previously determined in the case of $\mathrm{MbO}_{2}$ and assigned to the cooling of the $6 \mathrm{c}$ heme [26]. The third time constant of $\tau_{3}$, which was not accurately determined in this experiment due to the limited time window of $1.9 \mathrm{ps}$, can most likely be assigned to the fast geminate recombination phase or a few ps vibrational cooling of photolyzed hemoglobin that has been found in the case of $\mathrm{MbO}_{2}$ to have a time constant about 4 ps [26].

Broad spectral width of visible laser pulse enabled us to observe the pump-probe signal with a broadband range. A broadband multi-channel detector array was used to obtain the pump-probe signal at all of the probe frequencies simultaneously. In the simultaneous measurement at many probe wavelengths, we could obtain ultrafast spectral change after the photo-excitation of oxy-Hb only with a short measurement time, avoiding laser damage on the sample. Thus, we obtained the spectral changes in the ultrafast photo-dissociation process. The difference absorption spectra in the delay region between 0 and $100 \mathrm{fs}$ were averaged over 5 fs to observe the ultrafast spectral change. The observed difference absorption spectra have a positive sign, therefore they are thought to be due to induced absorption of photoexcited $\mathrm{HbO}_{2}$. The ultrafast spectral change shows oscillatory motion, which reflects wavepacket motion in the potential energy surface of the photoexcited $\mathrm{HbO}_{2}$ during the ultrafast photodissociation process. 


\section{Conclusion}

In this work, ultrafast time-resolved pump-probe signal was observed in visible spectral range using an ultrashort visible laser pulse. Generated visible laser pulse has a broadband spectrum, which enabled us to observe the pump-probe signal in broadband. Furthermore, the pump-probe signal could be obtained at all of the probe frequencies at the same time using a broadband multi-channel detector arrays. The simultaneous measurement at many probe wavelengths enables us to obtain ultrafast spectral change after the photo-excitation of oxy-hemoglobin only with a short measurement time, which helps a lot to avoid laser damage on the sample. Using the broadband visible ultrashort laser pulse together with the broadband multi-channel detector array, time-resolved difference absorption spectra were observed in a broadband probe spectral region extending from 523 to $719 \mathrm{~nm}$.

Exponential fitting for the observed time traces determined the primary-process time constant, of which upper limit value only was previously estimated. Ultrafast spectral change found in the delay region between 0 and $100 \mathrm{fs}$ is thought to be reflecting ultrafast photo-dissociation process of oxyhemoglobin.

\section{References}

[1] R.R. Anderson and A. Parrish, J. Invest. Dermatol. 77 (1981), 13-19.

[2] J.M. Baldwin and C. Chothia, J. Mol. Biol. 129 (1979), 175-200.

[3] D.A. Chernoff, R.M. Hochstrasser and A.W. Steele, Proc. Natl. Acad. Sci. USA 77 (1980), 5606-5610.

[4] P.A. Cornelius, R.M. Hochstrasser and A.W. Steele, J. Mol. Biol. 163 (1983), 119-128.

[5] S. Franzen, B. Bohn, C. Poyart, G. DePillis, S.G. Boxer and J.L. Martin, J. Biol. Chem. 270 (1995), 1718-1720.

[6] S. Franzen, B. Bohn, C. Poyart and J.L. Martin, Biochem. 34 (1995), 1224-1237.

[7] S. Franzen, L. Kiger, C. Poyart and J.L. Martin, Biophys. J. 80 (2001), 2372-2385.

[8] J.M. Friedman, T.W. Scott, G.J. Fisanick, S.R. Simon, E.W. Findsen, M.R. Ondrias and V.W. Macdonald, Science 229 (1985), 187-190.

[9] B.I. Greene, R.M. Hochstrasser, R.B. Weisman and W.A. Eaton, Proc. Natl. Acad. Sci. USA 75 (1978), 5255-5259.

[10] D. Houde, J.W. Petrich, O.L. Rojas, C. Poyart, A. Antonetti and J.L. Martin, in: Ultrafast Phenomena, Vol. V, G.R. Fleming and A.E. Siegman, eds, 1986, p. 419.

[11] T. Kobayashi, T. Saito and H. Ohtani, Nature 414 (2001), 531-534.

[12] T. Kobayashi, Z. Wang and T. Otsubo, J. Phys. Chem. A 111 (2007), 12985-12994.

[13] J.L. Martin, A. Migus, C. Poyart, Y. Lecarpentier, R. Astier and A. Antonetti, Proc. Natl. Acad. Sci. USA 80 (1983), 173-177.

[14] J.L. Martin, A. Migus, C. Poyart, Y. Lecarpentier, R. Astier and A. Antonetti, EMBO J. 2 (1983), 1815-1819.

[15] J.L. Martin, A. Migus, C. Poyart, Y. Lecarpentier and A. Antonetti, in: Hemoglobin, A.G. Schnek and C. Paul, eds, 1984.

[16] J.L. Martin, A. Migus, C. Poyart, Y. Lecarpentier, R. Astier and A. Antonetti, in: Ultrafast Phenomena, Vol. IV, D.H. Auston and K.B. Eisenthal, eds, 1984, p. 447.

[17] D.W. McCamant, P. Kukura and R.A. Mathies, Appl. Spectrosc. 57 (2003), 1317-1323.

[18] Y. Mizutani and T. Kitagawa, J. Phys. Chem. B 105 (2001), 10992-10999.

[19] M.F. Perutz, Ann. Rev. Biochem. 48 (1979), 327-386.

[20] J.W. Petrich, C. Poyart and J.L. Martin, Biochem. 27 (1988), 4049-4060.

[21] V.I. Prokhorenko, A.M. Nagy, S.A. Waschuk, L.S. Brown, R.R. Birge and R.J.D. Miller, Science 313 (2006), $1257-1261$.

[22] T.S. Rose, M.J. Rosker and A.H. Zewail, J. Chem. Phys. 88 (1988), 6672-6673.

[23] F. Schotte, J. Soman, J.S. Olson, M. Wulff and P.A. Anfinrud, J. Struct. Biol. 147 (2004), 235-246.

[24] R. Srinivasan, J.S. Feenstra, S.T. Park, S. Xu and A.H. Zewail, Science 307 (2005), 558-563.

[25] A. Waleh and G.H. Loew, J. Am. Chem. Soc. 104 (1982), 2346-2351.

[26] X. Ye, A. Demidov and P.M. Champion, J. Am. Chem. Soc. 124 (2002), 5914-5924.

[27] X. Ye, A. Demidov, F. Rosca, W. Wang, A. Kumar, D. Ionascu, L. Zhu, D. Barrick, D. Wharton and P.M. Champion, J. Phys. Chem. 107 (2003), 8156-8165. 


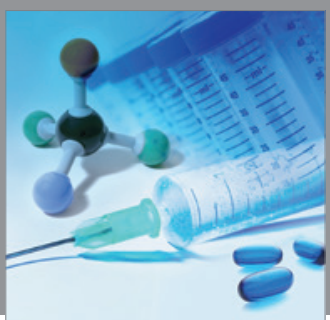

International Journal of

Medicinal Chemistry

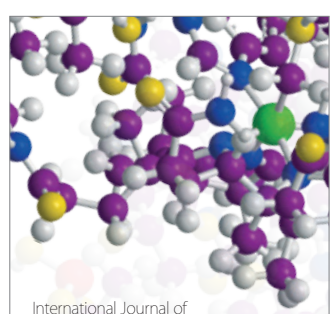

Carbohydrate Chemistry

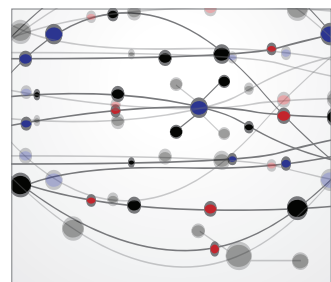

The Scientific World Journal
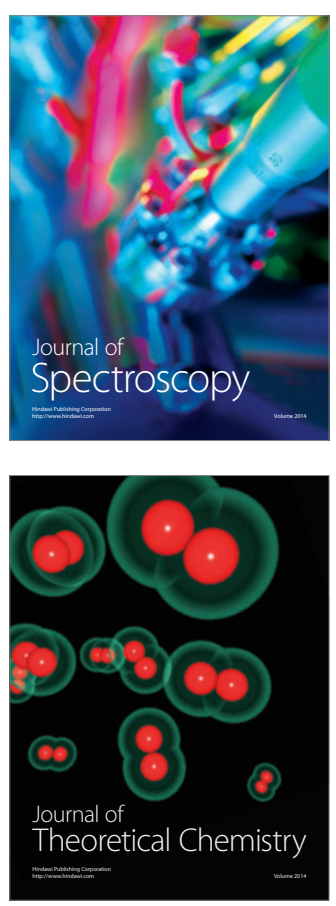
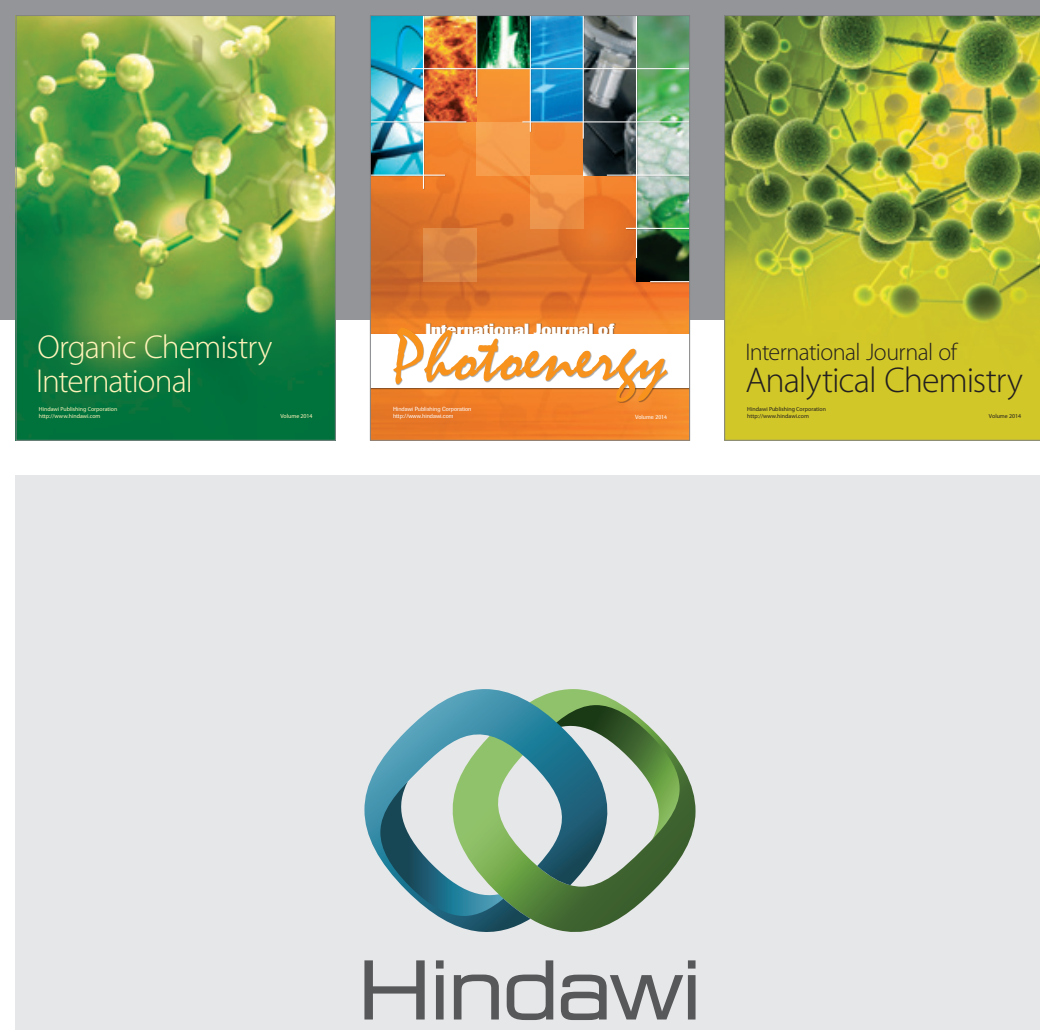

Submit your manuscripts at

http://www.hindawi.com
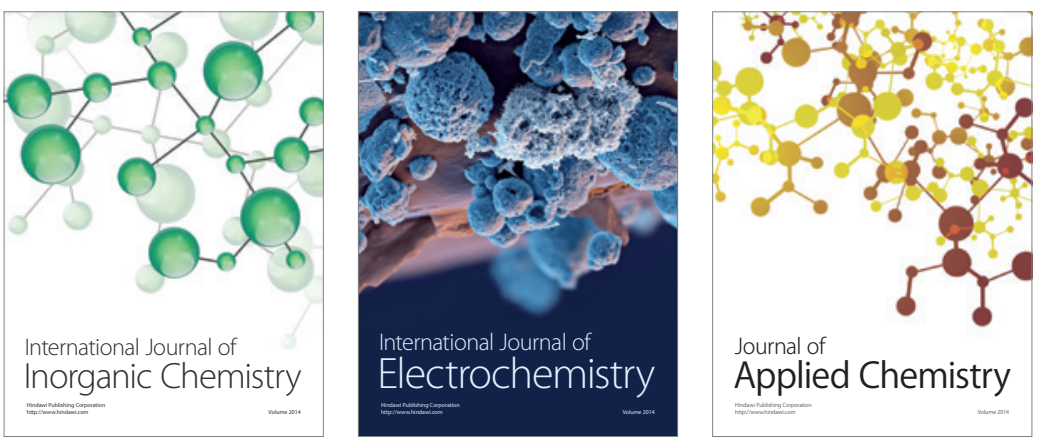

Journal of

Applied Chemistry
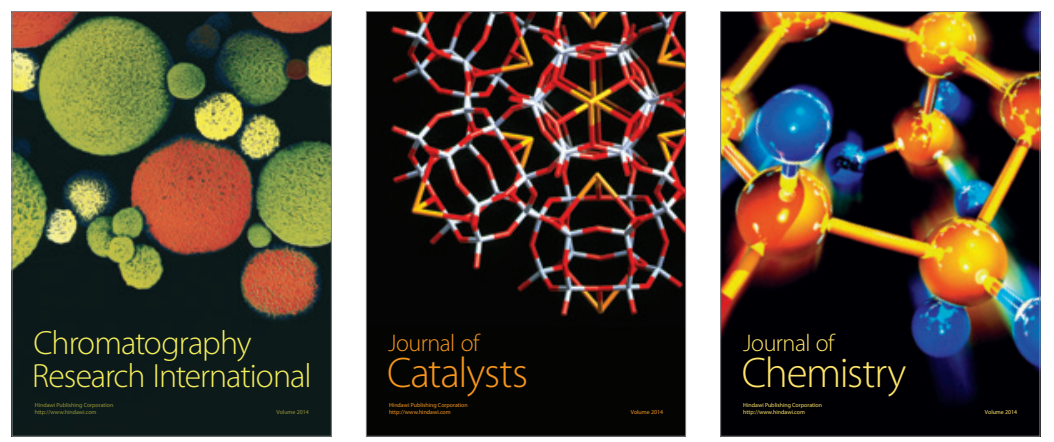
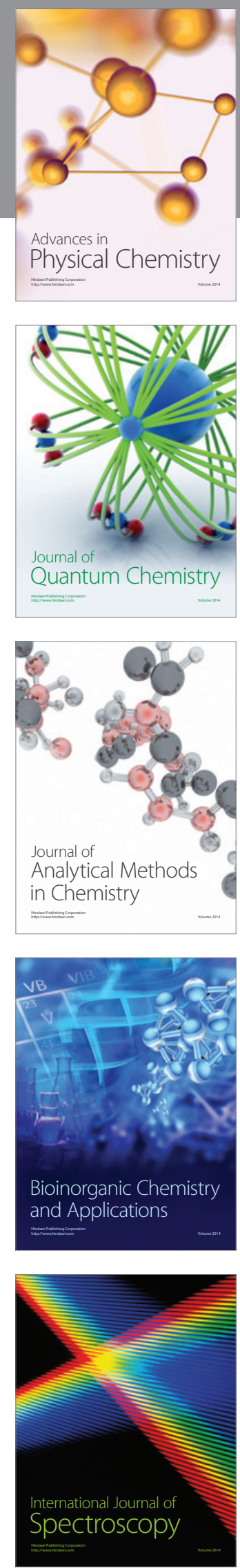